\title{
Networks analysis of genes and microRNAs in human Wilms' tumors
}

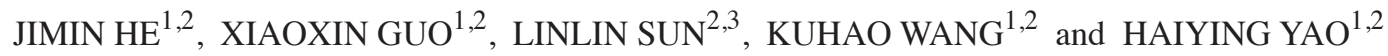 \\ ${ }^{1}$ College of Computer Science and Technology; \\ ${ }^{2}$ Key Laboratory of Symbolic Computation and Knowledge Engineering of the Ministry of Education; \\ ${ }^{3}$ College of Software, Jilin University, Changchun, Jilin 130012, P.R. China
}

Received January 15, 2015; Accepted February 12, 2016

DOI: $10.3892 / \mathrm{ol} .2016 .5102$

\begin{abstract}
Wilms' tumor (WT) is a common kidney cancer. To date, the expression of genes [transcription factors (TFs), target genes and host genes] and microRNAs (miRNAs/miRs) in WTs has captured the attention of biologists, while the regulatory association between the genes and miRNAs remains unclear. In the present study, TFs, miRNAs, target genes and host genes were considered as key factors in the construction of three levels of regulatory networks, namely, the differentially-expressed network, the related network and the global network. The four factors had three types of association, including the regulation of miRNAs by TFs, the targeting of the target genes by miRNAs and the location of miRNAs at host genes. The differentially-expressed network is the most important of the three networks, and only involves the differentially-expressed genes and miRNAs; with the exception of host genes, those elements all behave abnormally when a WT occurs, which suggests that the differentially-expressed network can accurately reveal the pathogenesis of WTs. E2F3, for example, is overexpressed in WTs, and it regulates hsa-let-7a, hsa-let-7a-1, hsa-miR-106b, among others. Meanwhile, E2F3 is targeted by hsa-miR-106b, hsa-miR-17 and hsa-miR-20a. If the regulatory network can be used to adjust those factors to a normal level, there may be a chance to cure patients with WTs. WT-associated factors were placed into the related network; this network is useful for understanding the regulatory pathways of genes and miRNA in WTs. The networks provide a novel perspective in order to
\end{abstract}

Correspondence to: Professor Xiaoxin Guo, College of Computer Science and Technology, Jilin University, 2699 Qianjin Street, Changchun, Jilin 130012, P.R. China

E-mail: guoxx@jlu.edu.cn

Abbreviations: WT, wilms' tumor; miRNA, microRNA; TF, transcription factor; NCBI, National Center for Biotechnology Information; TFBS, transcription factor binding site; FFL, feed-forward loop

Key words: Wilms' tumor, transcriptional factor, miRNA, target gene, host gene, network study the inner interactions of genes and miRNAs. The present study provides authoritative data and regulatory pathway analysis in order to partially elucidate the pathogenesis of WT, and thus supplies biologists with a basis for future research.

\section{Introduction}

Wilms' tumor (WT), also known as nephroblastoma, is the most common pediatric renal tumor, arising mainly in children $<5$ years of age. The tumor is believed to have an embryonic kidney cell origin (1). The current treatment protocol for WTs, as indicated by the International Society of Pediatric Oncology, involves the use of pre-operative chemotherapy followed by nephrectomy, however, such chemotherapy is not of equal benefit to all patients (2). It has been noted that differentially-expressed genes and microRNA (miRNA/miR) have a large impact on the development and therapy of WTs, and that associated genes and miRNA have less effect on WTs.

Genes include transcription factors (TFs), target genes and host genes, which play different roles in the network process. TFs and miRNAs are prominent gene expression regulators; gene regulatory factors are mainly formed from TFs and miRNAs, which control the developmental processes of genomic information in multicellular genomes by specifying when and where the genes are to be expressed $(3,4)$. Camas et al indicated that TFs are proteins that bind specific short DNA sequences adjacent to the genes whose transcription they regulate (5). miRNAs are a class of small non-coding RNA sequences that contribute to cancer development and metastasis.

miRNAs pair to the messages of protein-coding genes to target these genes for post-transcriptional repression (6).

miRNA locates at host genes. Rodriguez et al suggested that the transcriptional patterns of all miRNA host genes were selected from a range of sources, highlighting the spatial, temporal and physiological regulation of miRNA expression, which strongly suggested parallel transcription of the miRNA and the host transcripts, and the fact that the two differing miRNA transcription classes (exonic and intronic) may require slightly different biogenesis mechanisms (7).

A large amount of data on genes and miRNAs has been obtained from the experimentation of biologists and scientists. However, the majority of the experiments were based on one factor (gene or miRNA), and the regulatory association among 
the data remains unclear. In order to research WTs from a global perspective, the present study focused on the networks of TFs, miRNAs, target genes and host genes, and their control mechanisms. The differentially-expressed network involves differentially-expressed genes and miRNAs in the development and progression of WTs; the host genes where differentially-expressed miRNAs locate were considered as differentially expressed. The related network contains the associated genes and miRNAs in WT. The association between the genes and miRNA was analyzed in detail, comparing the similarities and differences. The vital linkages, in particular, were extracted for selective analysis. The study aimed to provide a greater understanding with regard to the pathogenesis of WTs.

\section{Materials and methods}

Extraction of TFs and miRNA regulatory associations. An experimentally validated dataset on TFs and the miRNAs that are regulated by them were gathered from TransmiR (8). The TransmiR dataset is a manually collated collection of regulatory associations between TFs and miRNAs. This complete data was considered as dataset S1.

Extraction of miRNAs and target gene regulatory associations. An experimentally validated dataset on miRNAs and their target genes was gathered from Tarbase5.0 (http://diana.imis. athena-innovation.gr/DianaTools/index.php?r=tarbase/index) and miRTarBase (http://mirtarbase.mbc.nctu.edu.tw/). To unify symbols, the official National Center for Biotechnology Information (NCBI) symbols of genes and miRNAs were used. The complete dataset was considered as dataset $\mathrm{S} 2$.

Extraction of miRNAs and host gene regulatory relations. miRNAs and their host genes were collected from the NCBI and miRBase (9). Official NCBI symbols were used for miRNAs and host genes. This dataset was regarded as dataset S3.

Extracting data of genes and miRNAs. Differentially-expressed miRNAs and associated miRNAs with regard to WT were gathered from the pertinent literature. The differentially-expressed miRNAs and associated miRNAs were considered as dataset $\mathrm{S} 4$ and S5, respectively.

Differentially-expressed genes were manually collected from the NCBI Single Nucleotide Polymorphism database (http://www.ncbi.nlm.nih.gov/snp/) and from the pertinent literature. Related genes were gathered from the GeneCards database (http://www.genecards.org/) and from the pertinent literature in the Web of Science (http://wokinfo.com/). The related genes were those to take part in the development, progression, radical therapy and clinical outcome of WT. In addition, P-match algorithm (10) was used to extract predicted TFs. The University of California Santa Cruz database (11) indicates that target genes of differentially-expressed miRNAs have $1,000 \mathrm{nt}$ promoter region sequences. P-match combining pattern and weight matrix approaches was used to identify TF binding sites (TFBSs) in 1,000- and 5,000-nt promoter region sequences, and the TFBSs were mapped onto the promoter regions of targets. The matrix library of P-match, as well as sets of known TFBSs collected in TRANSFAC (www.gene-regulation.com/pub/databases.html) provide the possibility of searching for a large variety of different TFBSs. Differentially-expressed genes and related genes were placed into the S6 and S7 datasets separately.

Three levels of network construction. All the regulatory associations in three levels of network (the differentially-expressed network, the related network and the global network) are based on TFs, miRNAs, target genes and host genes. Specifically, the datasets of S1, S2 and S3 contain all the associations among them, and furthermore, the global network is the combination of the three datasets.

The related network is a network that contains related genes and related miRNAs, and the regulatory relations between them. This was obtained by mapping S4 and S6 onto S1, S2 and S3.

The differentially-expressed network is the smallest and most important of the three networks. The factors it contains are differentially expressed. Differentially-expressed genes and miRNAs acted as primary factors to build up the network. The regulatory pathways were obtained by mapping S5 and S7 onto $\mathrm{S} 1, \mathrm{~S} 2$ and $\mathrm{S} 3$.

\section{Results}

Differentially-expressed network of WTs. Numerous significant regulatory pathways and factors are shown in Fig. 1; 5 TFs (E2F3, MYCN, SIX1, TP53 and CTNNB1), 38 differentially-expressed miRNAs, 33 host genes and 18 target genes (TFs targeted by miRNAs were also regarded as target genes) were determined. A number of regulatory pathways were highlighted in the complex network.

The associations of factors can be classified into three classes: i) miRNAs are regulated by TFs; ii) miRNAs target the target genes; and iii) miRNAs locate at host genes. The network is so complex for the following reasons: Firstly, certain TFs also act as target genes. For example, TP53 regulates hsa-miR-145, hsa-miR-192, hsa-miR-194, hsa-miR-215, hsa-miR-200a, hsa-miR-200b and hsa-miR-200c, but is also targeted by hsa-miR-25 and hsa-miR-125a-5p. Secondly, miRNAs and genes may form feed-forward loops (FFLs); for example, hsa-miR-106b targets E2F3, but is also regulated by E2F3. Thirdly, a single miRNA can locate at more than one host gene, such as hsa-let-7c, which locates at MIRLET7C, LINC00478 and C21orf34. Multiple different miRNAs can also locate at one host gene; for example, hsa-miR-19a, hsa-miR-17 and hsa-miR-20 are all located at C13orf26. In addition, IGF2 is overexpressed in WT (12). However, IGF2 acts as a target gene and a host gene; hsa-let-7a targets IGF2 and hsa-miR-483 locates at IGF2. Host genes together with their miRNAs assist in our comprehension of the development of WTs.

Associations among genes can be summarized as follows: Genes affect other genes through miRNAs. For instance, hsa-miR-145 targets IGF1R and is also regulated by TP53. This indicates that TP53 could produce an effect on IGF1R through hsa-miR-145. Combined experimentally validated factors and their actions reveal the pathogenesis of WTs.

Transcriptional network of TFs and miRNAs in WT. The transcriptional network is part of a differentially-expressed network. TFs are proteins that regulate miRNAs. The present 


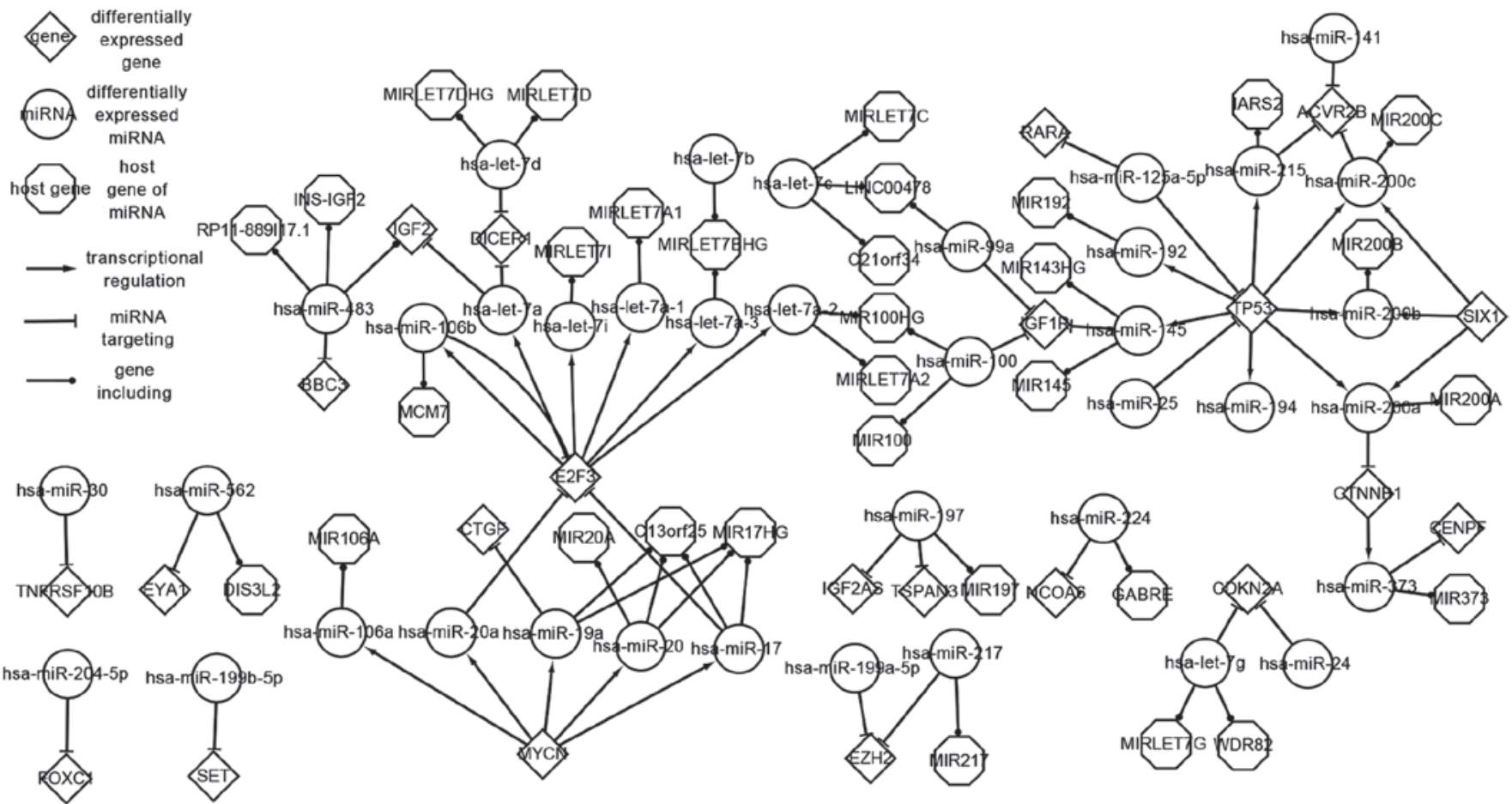

Figure 1. Differentially-expressed network of genes and miRNAs in Wilms' tumors. miR/miRNA, microRNA.

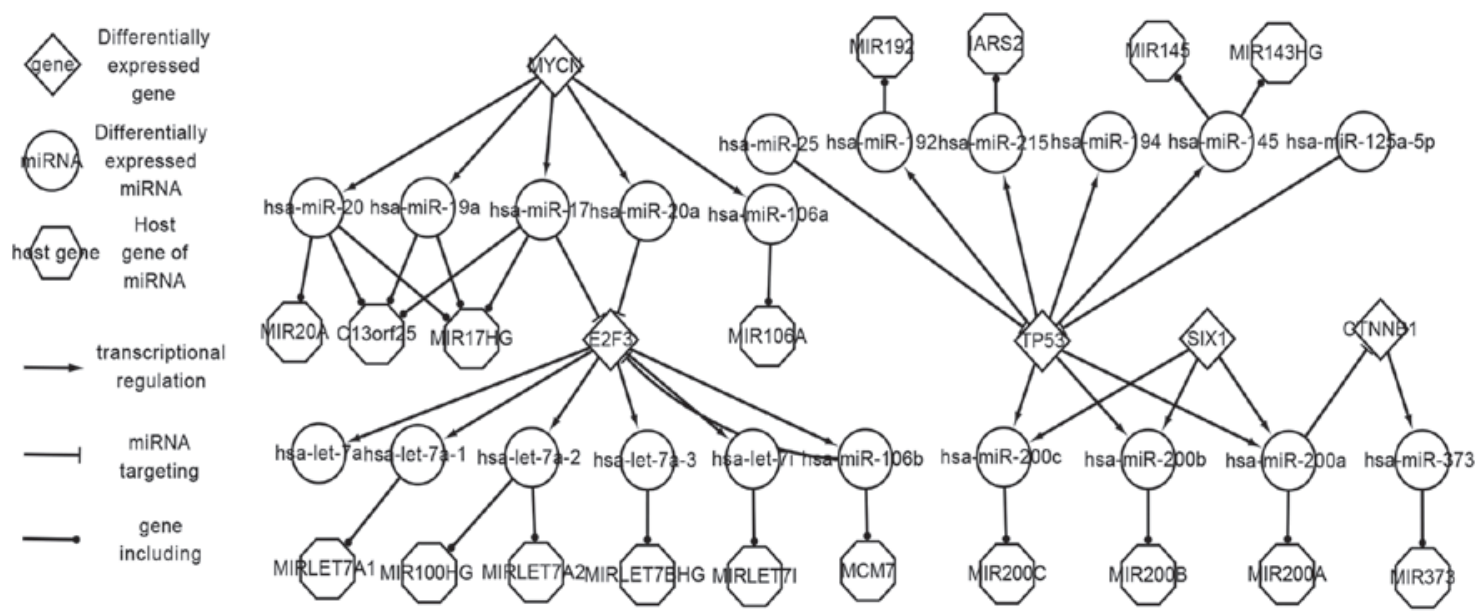

Figure 2. Transcriptional network of transcription factors and miRNAs in Wilms' tumors. miR/miRNA, microRNA.

study focused upon TFs, miRNAs and the regulatory associations between them. In total, 5 TFs (E2F3, TP53, SIX1, CTNNB1 and MYCN) play a vital role in WTs (Fig. 2).

E2F3 is the core factor in the transcriptional network. Cooper et al indicated that the E2F3 TF has an established role in controlling cell cycle progression and proliferation (13). Furthermore, E2F3 is overexpressed in WTs, implying that it may be a therapeutic target against the tumors (14). Hsa-miR-106b, hsa-miR-17 and hsa-miR-20a target E2F3, and E2F3 regulates 6 miRNAs (hsa-miR-106b, hsa-let-7a, has-let-7i, has-let-7a-1, hsa-let-7a-2 and hsa-let-7a-3). In other words, these upstream miRNAs affect downstream miRNAs through E2F3, which means that certain TFs can be regarded as bridges to connect predecessors and successors of miRNAs, and the expression of miRNAs in the important regulatory pathways when they have changed. E2F3 is such a vital factor that it should be a critical focus of attention.

SIX1 has certain differences to E2F3. SIX1 has been shown to be mainly highly expressed in the blastemal tissue components of WT (15). SIX1 was included in the network with other TFs in order to analyze WT in a systematic manner. No differentially-expressed miRNAs target SIX1, and SIX1 regulates hsa-miR-200a, hsa-miR-200b and hsa-miR-200c, which indicates that it may be the first factor to affect the expression of miRNAs in this regulatory pathway.

Related network of WTs. Numerous regulatory associations are shown in Fig. 3. The data in the related network include associated genes and associated miRNAs, including differentially-expressed genes and miRNAs. The related genes 


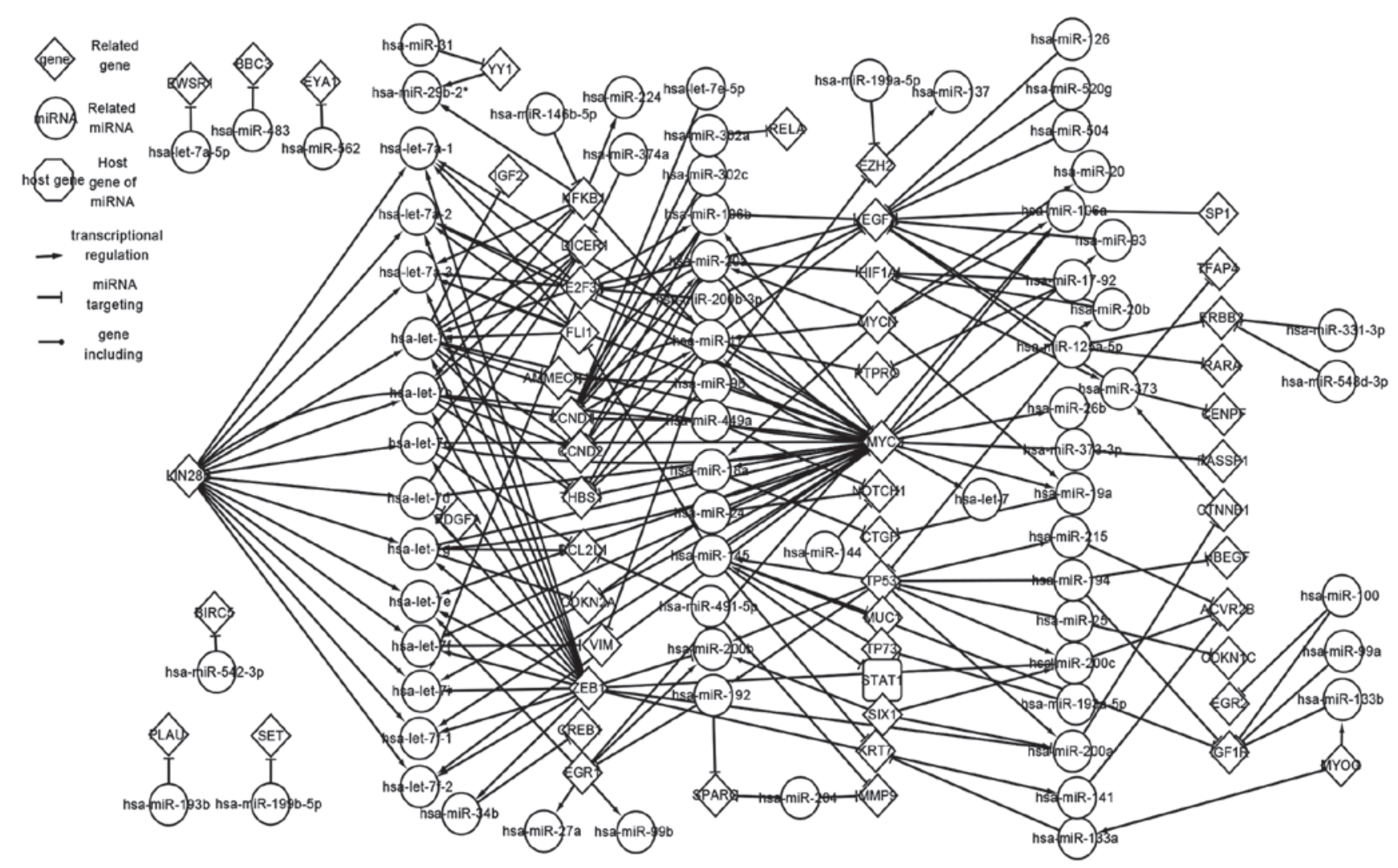

Figure 3. Related network of genes and miRNAs of Wilms' tumors. miR/miRNA, microRNA.

and miRNAs that have no associations with other factors are not shown in the figure. The present study focused on analyzing the pathways between the genes and miRNAs. The differentially-expressed network is clearly contained within the related network. Fig. 3 lists 18 TFs (such as LIN28B, MYC and MYCN), including five differentially-expressed TFs and three predicted TFs (ZEB1, NFKB1 and YY1), as well as 64 miRNAs (such as hsa-miR-100, hsa-miR-125 and hsa-miR-106a).

The related TFs LIB28B and MYC play prominent roles in the related network; they have the most regulatory associations. LIN28B regulates 13 miRNAs, including hsa-let-7a, hsa-let-7b and hsa-let-7c, and these miRNAs target certain other target genes, which means that LIN28B affects other target genes by regulating miRNAs. It is significant that LIN28B is targeted by no miRNAs. MYC regulates 23 related miRNAs, including hsa-let-7a, hsa-let-7b, hsa-let-7c and hsa-miR-19a, and is also targeted by 9 related miRNAs, including hsa-let-7a, hsa-let-7c and hsa-let-7g. The STAT1 gene is represented using a unique square; this gene was obtained from GeneCards and P-match.

ZEB1, NFKB1 and YY1 are three related TFs that were obtained from P-match. ZEB1 is targeted by 5 miRNAs (hsa-miR-200a, hsa-miR-200b, hsa-miR-200c, hsa-miR-141 and hsa-miR-429) and regulates 19 miRNAs (including hsa-let-7a, hsa-let-7b, hsa-let-7c, hsa-miR-200a, hsa-miR-200b and hsa-miR-200c). The pathways concerning ZEB1 are suggested to play a significant role in the development and pathogenesis of WTs. Detailed information on ZEB1, NFKB1 and YY1 is introduced in the following text.
Global network of WTs. The global network is too complex and large to be represented in this study. It contains a large number of associations between S1, S2 and S3 datasets. Certain genes and miRNAs in the global network are also involved with other tumors, such as renal cell carcinoma (16), which indicates that the global network affects more than one type of tumor. Therefore, the global network provides a novel perspective for research regarding the pathogenesis of WT.

Comparison and analysis of features of differentiallyexpressed genes in the WT networks. The pathways of the genes and miRNAs has been described as aforementioned; in this section, the study focuses on differentially-expressed genes. The total 84 differentially-expressed genes concerned with WT can be classified into 4 classes.

The first class of genes (E2F3, CTNNB1 and TP53) has 6 types of adjacency nodes. Those adjacency nodes are viewed as 3 successors and 3 predecessors. The following text focuses on E2F3.

Table I shows the miRNAs that target E2F3 and are regulated by E2F3. In total, 3 differentially-expressed miRNAs (hsa-miR-17, hsa-miR-20a and hsa-miR-106b) target E2F3 in the differentially-expressed network, while 4 and 11 miRNAs target E2F3 in the related and global networks, respectively. E2F3 regulates 6 differentially-expressed miRNAs (hsa-let-7a, hsa-let-7a-1, hsa-let-7a-2, hsa-let-7a-3, hsa-let-7i and hsa-miR-106b) in the differentially-expressed network. While in the related and global networks, E2F3 regulates 6 and 13 miRNAs, respectively. 
Table I. Regulatory association between E2F3 and miRNAs.

\begin{tabular}{|c|c|c|c|}
\hline Gene association & Differentially-expressed network & Related network & Global network \\
\hline \multicolumn{4}{|l|}{$\mathrm{E} 2 \mathrm{~F} 3$} \\
\hline miRNAs targeting the gene & miR-17, miR-20a, miR-106b & $\begin{array}{l}\text { miR-17, miR-20a, } \\
\text { miR-106b, miR-200b-3p }\end{array}$ & $\begin{array}{l}\text { miR-17, miR-20a, } \\
\text { miR-106b, miR-200b-3p, } \\
\text { miR-125b, miR-128, } \\
\text { miR-195, miR-203a, } \\
\text { miR-210, miR-34a, } \\
\text { miR-34c-5p }\end{array}$ \\
\hline miRNAs regulated by the gene & $\begin{array}{l}\text { let-7a, let-7a-1, let-7a-2, } \\
\text { let-7a-3, let-7i, miR-106b }\end{array}$ & $\begin{array}{l}\text { let-7a, let-7a-1, let-7a-2 } \\
\text { let-7a-3, let-7i, miR-106b }\end{array}$ & $\begin{array}{l}\text { let-7a, let-7a-1, let-7a-2, } \\
\text { let-7a-3, let-7i, } \\
\text { miR-106b, miR-15a, } \\
\text { miR-15b, miR-16, } \\
\text { miR-16-2, miR-195, } \\
\text { miR-34, miR-34a }\end{array}$ \\
\hline
\end{tabular}

miR/miRNA, microRNA.

The second class of genes includes EZH2 only. EZH2 has three successors and two predecessors. Differentially-expressed miRNAs hsa-miR-199a-5p and hsa-miR-217 target EZH2 in the differentially-expressed network, while 4 and 16 miRNAs target EZH2 in the related and global networks, respectively. No differentially-expressed miRNAs are regulated by EZH2 in the differentially-expressed network. Related miRNA hsa-miR-137 is regulated by EZH2 in the related network and 3 miRNAs are regulated by EZH2 in the global network.

The third class of genes (TP73, MYCN, SIX1 and EGFR) has 4 types of adjacency nodes. Despite the fact that they all have 4 adjacency nodes, these genes are not the same. TP73 has no adjacency node in the differentially-expressed network. TP73 regulates hsa-miR-145 and is targeted by hsa-miR-193a-5p in the related network. MYCN and SIX1 are targeted by no miRNAs in the differentially-expressed and related networks. EGFR regulates no miRNAs in the differentially-expressed and related networks.

The fourth class of genes (including DICER1, BBC3, RARA, IGF1R and ACVR2B) has 3 types of adjacency nodes. All of these differentially-expressed genes have no successor, but have 3 predecessors. For example, DICER1 is targeted by hsa-let-7a and hsa-let-7d in the differentially-expressed network, while 9 and 17 miRNAs target DICER1 in the related and global networks, respectively. No miRNA is regulated by DICER1 in the three levels of network.

Comparison and analysis offeatures of differentially-expressed miRNAs in the WT networks. The same method was used to compare every differentially-expressed miRNA, and 87 validated differentially-expressed miRNAs were extracted from the permanent literature. These can be classified into four classes.

The first class of miRNAs (hsa-miR-17, hsa-miR-19a, hsa-miR-20a, hsa-miR-145, hsa-miR-200c and hsa-miR-373) has 6 types of adjacency nodes. All 6 miRNAs have 3 successors and 3 predecessors. hsa-miR-20a is used here as an example to fully understand the regulatory mechanisms between genes and miRNAs (Table II). MYCN regulates hsa-miR-20a in the differentially-expressed network, 3 and 10 genes regulate hsa-miR-20a in the related and global networks, respectively. E2F3 is targeted by hsa-miR-20a in the differentially-expressed network. Furthermore, 7 and 29 genes are targeted by hsa-miR-20a in the related and global networks, respectively.

The second class of miRNAs (hsa-let-7d, hsa-let-7g and hsa-miR-106a) has 5 types of adjacency nodes. Despite the same amount of adjacency nodes, hsa-miR-106a has 2 successors and 3 predecessors, while the other miRNAs (hsa-let-7d and hsa-let-7g) have 3 successors and 2 predecessors. Hsa-miR106a is regulated by MYCN in the differentially-expressed network, while 4 and 6 genes regulate hsa-miR106a in the related and global networks, respectively. There is no miRNA targeted by hsa-miR106a in the differentially-expressed network. In the related network, VEGFA is targeted by hsa-miR106a. In addition, hsa-miR106a targets 22 genes.

The third class of miRNAs (hsa-miR-224, hsa-miR-25, hsa-miR-24, hsa-miR-192, hsa-miR-194 and hsa-miR-200b) has 4 types of adjacency nodes. While 3 of them (hsa-miR-224, hsa-miR-25 and hsa-miR-24) have 3 types of successors and 1 type of predecessor, the others have 1 type of successor and 3 types of predecessors. Hsa-miR-224 targets NCOA6 in the differentially-expressed and related networks, and 18 genes are targeted by hsa-miR-224 in the global network.

The fourth class of miRNAs (hsa-miR-126, hsa-miR-154, hsa-miR-197, hsa-miR-22, hsa-miR-27a and hsa-miR-302a) has 3 types of adjacency nodes. The 3 types of adjacency nodes can be regard as 2 types of 2 successors and 1 type of predecessor. For example, none of the genes regulate or are targeted by hsa-miR-126 in the differentially-expressed network; VEGFA is targeted by hsa-miR-126 in the related network.

Comparison and analysis of features of predicted TFs in the WT networks. In total, 17 predicted TFs (including NFKB1, ZEB1, CREB1, HLF, ELK, NR2F2, STAT1, CUX1 and YY1) were extracted by P-match, and the same method as aforementioned was used to analyze them. Among the 17 TFs, 
Table II. Regulatory relation between hsa-miR-20a and genes.

\begin{tabular}{|c|c|c|c|}
\hline Regulatory relation & Differentially-expressed network & Related network & Global network \\
\hline \multicolumn{4}{|l|}{ hsa-miR-20a } \\
\hline Genes that regulate miRNA & MYCN & $\begin{array}{l}\text { MYCN, CCND1, MYC } \\
\text { E2F1, ESR1, NKX2-5, } \\
\text { SPI1, STAT5B, TLX1, } \\
\text { TLX3 }\end{array}$ & MYCN, CCND1, MYC, \\
\hline Genes targeted by miRNA & $\mathrm{E} 2 \mathrm{~F} 3$ & $\begin{array}{l}\text { E2F3, CCND1, CCND2 } \\
\text { HIF1A, MYC, THBS1 } \\
\text { VEGFA }\end{array}$ & $\begin{array}{l}\text { E2F3, CCND1, CCND2, } \\
\text { HIF1A, MYC, THBS1, } \\
\text { VEGFA, APP, BCL2, } \\
\text { BIM, BMPR2, BNIP2, } \\
\text { CDKN1A, E2F1, IL8, } \\
\text { JAK1, MAP3K12, MAPK9, } \\
\text { MEF2D, MUC17, NRAS, } \\
\text { PTEN, RB1, RBL1, RBL2, } \\
\text { RUNX1, WEE1, SMAD4, } \\
\text { TGFBR2 }\end{array}$ \\
\hline
\end{tabular}

miR/miRNA, microRNA.

Table III. Regulatory relation between ZEB1 and miRNAs.

\begin{tabular}{|c|c|c|c|}
\hline Regulatory relation & Differentially-expressed network & Related network & Global network \\
\hline \multicolumn{4}{|l|}{ ZEBI } \\
\hline miRNAs targeting the gene & $\begin{array}{l}\text { miR-200a, miR-200b, } \\
\text { miR-200c, miR-141 }\end{array}$ & $\begin{array}{l}\text { miR-200a, miR-200b } \\
\text { miR-200c, miR-141 }\end{array}$ & $\begin{array}{l}\text { miR-200a, miR-200b, } \\
\text { miR-200c, miR-141, } \\
\text { miR-205, miR-429 }\end{array}$ \\
\hline miRNAs regulated by the gene & $\begin{array}{l}\text { let-7, let-7a, let-7a-1, } \\
\text { let-7a-2, let-7a-3, let-7b, } \\
\text { let-7c, let-7d, let-7e, } \\
\text { let-7f, let-7f-1, let-7f-2, } \\
\text { let-7g, miR-141, miR-200a, } \\
\text { miR-200b, miR-200c }\end{array}$ & $\begin{array}{l}\text { let-7, let-7a, let-7a-1, } \\
\text { let-7a-2, let-7a-3, let-7b, } \\
\text { let-7c, let-7d, let-7e, } \\
\text { let-7f, let-7f-1, let-7f-2, } \\
\text { let-7g, miR-141, miR-200a, } \\
\text { miR-200b, miR-200c, } \\
\text { miR-34b }\end{array}$ & $\begin{array}{l}\text { let-7, let-7a, let-7a-1, } \\
\text { let-7a-2, let-7a-3, let-7b, } \\
\text { let-7c, let-7d, let-7e, } \\
\text { let-7f, let-7f-1, let-7f-2, } \\
\text { let-7g, miR-141, } \\
\text { miR-200a, miR-200b, } \\
\text { miR-200c, miR-34b, } \\
\text { miR-34, miR-34a }\end{array}$ \\
\hline
\end{tabular}

$\operatorname{miR} / \operatorname{miRNA}$, microRNA.

13 have no adjacency nodes in the differentially-expressed and related networks. Only NFKB1, ZEB1, STAT1 and YY1 were analyzed in this section. These genes can be classified into 2 types of genes.

The first type of TF (ZEB1 and NFKB1) has 6 types of adjacency nodes. Table III shows the regulatory mechanism for ZEB1. The hsa-miR-200a, hsa-miR-200b, hsa-miR-200c and hsa-miR-141 differentially-expressed miRNAs target ZEB1. In the related network, ZEB1 is also targeted by these 4 miRNAs. While 6 miRNAs target ZEB1 in the global network, 17 miRNAs (including hsa-let-7, hsa-let-7a, hsa-let-7a-1, hsa-let-7a-2 and hsa-let-7a-3) regulate ZEB1 in the differentially-expressed network. A total of 18 and 20 miRNAs regulate ZEB1 in the related and global networks, respectively.
The second class of TFs (YY1) has 4 types of adjacency nodes. Hsa-miR-29b-2 is regulated by YY1 in the related network; and 8 miRNAs are regulated by YY1 in the global network. Hsa-miR-31 targets YY1 in the related network, and 2 miRNAs (hsa-miR-31 and hsa-miR-34a) target YY1 in the global network.

\section{Discussion}

In the present study, validated data were collected from authoritative databases and the permanent literature. These data were composed of genes and miRNAs. Three levels of network (the differentially-expressed network, the related network and the global network) were constructed from genes, miRNAs and the regulatory mechanisms between them. The 
intricate association between genes and miRNAs was the most significant point to analyze in the study.

After fully researching the networks and comparing the similarities and differences among the pathways, certain important factors and pathways were found. There are 5 TFs (E2F3, MYCN, TP53, SIX1 and CTNNB1) in the differentially-expressed network. Notably, SIX1 regulates hsa-miR-200a, hsa-miR-200b and hsa-miR-200c in the differentially-expressed network. However, no miRNA targets SIX1 in the differentially-expressed and related networks, which suggests that SIX1 may be the first factor in the pathogenesis of WT. In addition, E2F3 together with hsa-miR-106b forms an FFL in the differentially-expressed network; furthermore, LIN28B and hsa-let-7d also form an FFL in the related network. These FFLs make a difference in the pathogenesis and progression of WT as they have been identified to serve a key role in increasing tumor growth and regulation of WT mechanisms (17). In order to investigate the pathogenesis and determine better methods for curing patients with WTs, further comprehensive and authoritative data and pathways will be used to construct the three level networks, which can be used to explain the interaction between genes and miRNAs more clearly.

The method proposed by the present study may be useful in preventing the occurrence of WT. Core genes and miRNAs not only affect WT, but exhibit an important function in other tumors. CTNNB1 exhibits a significant role in WT, while it is also involved in hepatoblastoma (18). Furthermore, hsa-let-7a is differentially expressed in WT, and is also overexpressed in retinoblastoma (19). The present study provides a novel perspective for biologists with regard to WT treatment. The same differentially-expressed genes and miRNAs exhibit various functions in different tumors. Therefore, future studies will focus on the similarities and differences among similar studies on other cancers.

\section{Acknowledgements}

This study was supported by the National Natural Science Foundation of China (grant no. 60905022).

\section{References}

1. Beckwith JB, Kiviat NB and Bonadio JF: Nephrogenic rests, nephroblastomatosis and the pathogenesis of Wilms' tumor. Pediatr Pathol 10: 1-36, 1990.
2. Watson JA, Bryan K, Williams R, Popov S, Vujanic G Coulomb A, Boccon-Gibod L, Graf N, Pritchard-Jones K and O'Sullivan M: miRNA Profiles as a predictor of chemoresponsiveness in Wilms' tumor blastema. PLoS One 8: e53417, 2013.

3. Hobert O: Gene regulatory by transcription factors and microRNAs. Science 319: W1785-W1786, 2008.

4. Levine M and Davidson EH: Gene regulatory networks for development. Sci USA 102: 4936-4942, 2005.

5. Camas FM, Alm EJ and Poyatos JF: Local gene regulation details a recognition code within the laci transcriptional factor family. PLoS Comput Biol 6: e1000989, 2010.

6. Bartel DP: MicroRNAs: Genomics, biogenesis, mechanism and function. Cell 116: 281-297, 2004.

7. Rodriguez A, Griffiths-Jones S, Ashurst JL and Bradley A: Identification of mammalian microRNA host genes and transcription units. Genome Res 14: 1902-1910, 2004.

8. Wang J, Lu M, Qiu C and Cui Q: TransmiR: A transcription factor-microRNA regulation database. Nucleic Acids Res 38: D119-D122, 2009.

9. Kozomara A and Griffiths-Jones S: miRBase integrating microRNA annotation and deep-sequencing data. Nucleic Acids Res 39: D152-D157, 2011.

10. Chekmenev DS, Haid C and Kel AE: P-Match: Transcription factor binding site search by combining patterns and weight matrices. Nucleic Acids Res 33: W432-W437, 2005.

11. Fujita PA, Rhead B, Zweig AS, Hinrichs AS, Karolchik D, Cline MS, Goldman M, Barber GP, Clawson H, Coelho A, et al: The UCSC genome browser database: Update. Nucleic Acids Res 39: D876-D882, 2011.

12. Zim B, Hartmann O, Samans B, Krause M, Wittmann S, Mertens F, Graf N, Eilers M and Gessler M: Expression profiling of Wilms tumors reveals new candidate genes for different clinical parameters. Int J Cancer 118: 1954-1962, 2006.

13. Cooper CS, Nicholson AG, Foster C, Dodson A, Edwards S, Fletcher A, Roe T, Clark J, Joshi A, Norman A, et al: Nuclear overexpression of the E2F3 transcription factor in human lung cancer. Lung Cancer 54: 155-162, 2006.

14. An Q, Wang Y, An R, Li Y, Yao T, Zhai B and Sun X: Association of E2F3 expression with clinicopathological features of Wilms' tumors. J Pediatr Surg 48: 2187-2193, 2013.

15. Sehic D, Karlsson J, Sandstedt B and Gisselsson D: SIX1 protein expression selectively identifies blastemal elements in Wilms tumor. Pediatr Blood Cancer 59: 62-68, 2012.

16. Song CL, Xu ZW, Jin Y, Zhu M, Wang K and Wang N: The network of microRNAs, transcription factors, target genes and host genes in human renal cell carcinoma. Oncol Lett 9: 498-506, 2015.

17. Wang L, Che XJ, Wang N, Li J and Zhu MH: Regulatory network analysis of microRNAs and genes in neuroblastoma. Asian Pac J Cancer Prev 10: 7645-7652, 2014.

18. Koch A, Denkhaus D, Albrecht S, Leuschner I, von Schweinitz D and Pietsch T: Childhood hepatoblastomas frequently carry a mutated degradation targeting box of the beta-catenin gene. Cancer Res 59: 269-273, 1999.

19. Li J, Xu ZW, Wang KH, Wang N, Li DQ and Wang S: Networks of microRNAs and genes in retinoblastomas. Asian Pac J Cancer Prev 14: 6631-6636, 2014. 